Article

\title{
Genetic Analysis of Peroxisomal Genes Required for Longevity in a Yeast Model of Citrin Deficiency
}

\author{
Chalongchai Chalermwat ${ }^{1, *}$, Thitipa Thosapornvichai ${ }^{2}$, Laran T. Jensen ${ }^{2} \mathbb{D}$ and \\ Duangrurdee Wattanasirichaigoon ${ }^{3}$ (D) \\ 1 Department of Biochemistry, Faculty of Medicine, Khon Kaen University, Khon Kaen 40002, Thailand \\ 2 Department of Biochemistry, Faculty of Science, Mahidol University, Bangkok 10400, Thailand; \\ pa_lim_piim@hotmail.com (T.T.); laran.jen@mahidol.ac.th (L.T.J.) \\ 3 Division of Medical Genetics, Department of Pediatrics, Faculty of Medicine Ramathibodi Hospital, Mahidol \\ University, Bangkok 10400, Thailand; duangrurdee.wat@mahidol.ac.th \\ * Correspondence: cchanl@kku.ac.th; Tel.: +66-4336-3265
}

Received: 25 November 2019; Accepted: 7 January 2020; Published: 9 January 2020

\begin{abstract}
Citrin is a liver-specific mitochondrial aspartate-glutamate carrier encoded by SLC25A13. Citrin deficiency caused by SLC25A13 mutation results in carbohydrate toxicity, citrullinemia type II, and fatty liver diseases, the mechanisms of some of which remain unknown. Citrin shows a functional homolog in yeast aspartate-glutamate carrier (Agc1p) and agc1 $\Delta$ yeasts are used as a model organism of citrin deficiency. Here, we found that agc1 $\Delta$ yeasts decreased fat utilization, impaired NADH balance in peroxisomes, and decreased chronological lifespan. The activation of GPD1-mediated $\mathrm{NAD}^{+}$regeneration in peroxisomes by GPD1 over-expression or activation of the malate-oxaloacetate NADH peroxisomal shuttle, by increasing flux in this NADH shuttle and over-expression of $M D H 3$, resulted in lifespan extension of $\operatorname{agc1} 1 \triangle$ yeasts. In addition, over-expression of PEX34 restored longevity of agc1 $\triangle$ yeasts as well as wild-type cells. The effect of PEX34-mediated longevity required the presence of the GPD1-mediated NADH peroxisomal shuttle, which was independent of the presence of the peroxisomal malate-oxaloacetate NADH shuttle and PEX34-induced peroxisome proliferation. These data confirm that impaired $\mathrm{NAD}^{+}$regeneration in peroxisomes is a key defect in the yeast model of citrin deficiency, and enhancing peroxisome function or inducing $\mathrm{NAD}^{+}$regeneration in peroxisomes is suggested for further study in patients' hepatocytes.
\end{abstract}

Keywords: citrin deficiency; mitochondrial aspartate-glutamate carrier; peroxisomes; $\mathrm{NAD}^{+}$ regeneration; Saccharomyces cerevisiae

\section{Introduction}

Citrin deficiency is an inherited non-alcoholic fatty liver disease commonly found in the Asia-Pacific population [1-3]. This disorder results from a loss-of-function mutation in SLC25A13 encoding a mitochondrial aspartate-glutamate carrier, called citrin or AGC2, that appears to have a primary role in the liver [4,5]. Citrin deficiency results in an impaired malate-aspartate shuttle and decreased $\mathrm{NAD}^{+}$regeneration in the cytosol leading to carbohydrate intolerance and type II citrullinemia (CTLN2) [6,7]. Current therapies for citrin deficiency are limited, although treatment with sodium pyruvate, arginine hydrochloride, and restriction of carbohydrate intake has shown promise in reducing symptoms $[7,8]$.

Saccharomyces cerevisiae (budding yeast) has been shown to be a suitable model system for the study of several human diseases, including citrin deficiency $[9,10]$. S. cerevisiae is a genetically tractable organism and can be used to search for genetic suppressors to identify functional relationships between genes that may not otherwise be identified via other techniques [11]. Yeast contains a homolog of 
citrin, $A G C 1$, and the deletion of this gene results in decreased growth in acetate and oleic media [12]. Ectopic expression of SLC25A13 in agc1 $\triangle$ yeasts can restore growth in acetate medium, demonstrating that yeast $A G C 1$ is functionally equivalent to human citrin [10,12].

In addition to mitochondria, peroxisomes can contribute to utilization of acetate and fatty acids [13-15]. Loss of $A G C 1$ in yeasts impairs growth in acetate and oleic media, but not in glycerol medium, suggesting that it likely affects peroxisomal rather than mitochondrial functions [10]. Peroxisomes are single-membrane-bound organelles derived from the endoplasmic reticulum (ER) and are found in eukaryotic cells including those of both yeasts and humans [16]. A major function of peroxisomes is to support mitochondrial metabolism through fatty acid oxidation as well as degradation of hydrogen peroxide, and these activities are induced in yeast following entry into the stationary phase of growth [16-18]. Survival of yeast in the stationary phase has been extensively utilized as a model of aging and is referred to as the chronological lifespan [19]. In this study, we report that agc1 $\Delta$ yeast have impaired peroxisomal $\mathrm{NAD}^{+}$regeneration, decreased fat utilization, and decreased chronological lifespan. Genetic analysis of $a g c 1 \Delta$ yeast has revealed that the induction of peroxisome functions relating to $\mathrm{NAD}^{+}$regeneration is capable of increasing the longevity of $\operatorname{agc1} 1 \Delta$ yeast. The reduced lifespan of human hepatocytes with a loss-of-function mutation in citrin may contribute to disease progression. Therefore, enhancing peroxisome function may be an alternative treatment for citrin deficiency.

\section{Materials and Methods}

\subsection{Primers and Plasmids}

The AGC1 disruption plasmid was generated by PCR amplifying upstream (-931 to -114$)$ and downstream sequences (+2499 to +3478 ) of ACG1 introducing BamHI and SalI (upstream) or NotI and BamHI (downstream) restriction sites. Following digestion, the AGC1 DNA fragments were inserted into pRS403 (HIS3) [20]. The expression plasmids including PEX34, PEX5, PEX11, MDH3, GPD1, and NDE2 expression plasmids were constructed using Ycplac33 (CEN URA3) vector, a yeast centromeric plasmid containing the URA3 selectable marker and the TPI1 promoter [21]. The NADH sensor plasmids were derived from pC1-REX-YFP, a gift from Vsevolod Belousov (Addgene plasmid \#48247). The REX-YFP fusion protein will interact with NADH and can emit fluorescent signals [22]. pC1-REX-YFP was introduced at $5^{\prime} \mathrm{XbaI}$ and NdeI sites by PCR mutagenesis and verified. The mutated pC1-REX-YFP was digested with XbaI and SacII to obtain the NADH sensor sequence. The backbone plasmid pLJ339 (PGK1 promoter, CEN, LEU2), a yeast centromeric plasmid containing the LEU2 selectable marker and the PGK1 promoter, was digested with the same enzymes. The NADH sensor sequence was then ligated to the isolated pLJ339 backbone plasmid. The cytosolic NADH sensor plasmid was selected and verified. To construct the peroxisomal NADH sensor plasmid, the NADH sensor sequence in the cytosolic NADH sensor plasmid was introduced a C-terminal peroxisomal targeting sequence by PCR mutagenesis. The cytosolic NADH sensor plasmid was selected and verified. The lists of primers used in the study is shown in Supplementary Table S1.

\subsection{Yeast Strains and Disruption of AGC1}

Yeast strains used in this study were derived from BY4742 [23]. Single-deletion yeast strains were obtained from Open Biosystems, Inc. (Waltham, MA, USA). The AGC1 gene was disrupted using the knockout plasmid, linearized by digestion with BamH1, and transformed into yeast strains. The AGC1 gene of yeast was replaced with the HIS3 selectable marker by homologous recombination. Correct plasmid integration and deletion of $A G C 1$ sequences was verified by in vivo PCR using flanking primers [24]. The lists of yeast strains and genotypes used in the study is shown in Supplementary Table S2. 


\subsection{Yeast Transformation}

The control plasmid (pRS316), expression plasmids, and linearized AGC1 disruption plasmids were transformed into specific yeast strains using a lithium acetate procedure [25]. Cells were propagated at $30^{\circ} \mathrm{C}$ either in enriched yeast extract, peptone-based medium (YPD), synthetic complete (SC), or synthetic deficient (SD) medium containing $2 \%$ glucose [26]. Yeast transformants were selected on SC medium lacking the appropriate nutrient.

\subsection{Culture Conditions and Longevity Assay}

Yeasts were grown on synthetic deficient (SD) plate supplemented with $2 \%$ glucose incubated in a $30{ }^{\circ} \mathrm{C}$ incubator for three days. Then, they were pre-cultured in synthetic SD broth containing $2 \%$ glucose by incubation at $30{ }^{\circ} \mathrm{C}$, with shaking at $220 \mathrm{rpm}$ for $24 \mathrm{~h}$. Cells were harvested and inoculated in SD broth with $2 \%$ glucose at a starting optical density $600 \mathrm{~nm}\left(\mathrm{OD}_{600}\right)$ of 0.1 and grown for nine days. The chronological longevity assay was used to determine survival in the stationary phase [19]. Cell survival was monitored at Day 3 and Day 9 of culture. Yeast cells were collected and washed twice with sterile distilled water. The $\mathrm{OD}_{600}$ was measured, and samples were prepared in sterile distilled water at $10^{5}, 10^{4}$, and $10^{3}$ cells. Yeasts $(10 \mu \mathrm{L}$ each) were dropped on an SD plate supplemented with $2 \%$ glucose, incubated at $30^{\circ} \mathrm{C}$ for $72 \mathrm{~h}$, and then imaged.

\subsection{Peroxisomal and Cytosolic NADH Analysis, Peroxisome Proliferation Analysis, and Confocal Fluorescence Microscopy}

To analyze peroxisomal and cytosolic NADH fluorescence signals, yeasts were grown on an SD plate supplemented with $2 \%$ glucose incubated in a $30{ }^{\circ} \mathrm{C}$ incubator for five days and then directly taken for visualizing NADH signals with confocal fluorescence microscopy. The excitation and emission wavelengths were chosen at $488 / 510 \mathrm{~nm}$. The small punctate green fluorescence represented peroxisomal NADH signals and the diffuse green fluorescence represented cytosolic NADH signals. To analyze peroxisome proliferation, yeasts were grown on an SD plate supplemented with $2 \%$ glucose for three days. Then, they were cultured in SD broth supplemented with $0.1 \%$ glucose until late log phase. The punctate signals from red fluorescent protein (RFP) fusions RFP-PTS1 (peroxisome marker) represented peroxisome numbers [27]. To visualize fluorescence signals derived from NADH sensor protein or RFP-PTS1 protein, the live cells were directly viewed at a magnification of 60× with an Olympus FV1000 confocal laser scanning microscope (Olympus Bioimaging Center, Mahidol University).

\subsection{Neutral Lipid Staining and Spectrofluorometry}

To analyze neutral lipids (esterified fatty acids), yeasts were precultured and cultured the same as for longevity assays. Cells were taken for staining neutral lipids with Nile red fluorescence dye at Day 3 and Day 9 of culture. One milliliter of cell volumes was collected, washed with phosphate buffered saline twice, and resuspended to $\mathrm{OD}_{600 \mathrm{~nm}} 1.0\left(2 \times 10^{4}\right.$ cell $\left./ \mu \mathrm{L}\right)$. Then, $250 \mu \mathrm{L}$ of cell volumes $(5$ $\times 10^{6}$ cells) was incubated with dimethyl sulfoxide: phosphate buffered saline 1:1 for 2 min in one well of a 96-well plate. Yeasts were then stained with $25 \mu \mathrm{L}$ of Nile red $(60 \mu \mathrm{g} / \mathrm{mL})$. The fluorescence signals in $5 \mu \mathrm{g} / \mathrm{mL}$ of Nile red were read for $20 \mathrm{~min}$ (read every $1 \mathrm{~min} \times 20$ cycles) during shaking at $180 \mathrm{rpm}$ at $30{ }^{\circ} \mathrm{C}$ in a spectrofluorometer machine. The excitation and emission wavelength were chosen at $485 / 535 \mathrm{~nm}$. The experiment was performed in triplicate, and the highest fluorescence intensity prior to plateau was chosen for analysis. The relative fluorescence intensity was corrected with background fluorescence intensity obtained from the control well. Neutral lipid contents in yeasts were presented as relative fluorescence units. 


\subsection{Data Analysis}

The difference in neutral lipid contents was analyzed by IBM SPSS statistics version 22. Statistical analyses within groups were done using paired-samples $t$ tests (paired $t$-test) and a significant difference was indicated when $p$-value $<0.05$. Yeasts with neutral lipid staining were derived in triplicate from one repeated experiment.

\section{Results}

\subsection{Deletion of AGC1 Inhibits Fat Utilization in the Stationary Phase}

Fatty acid is esterified and stored in fat droplets in cytosol as an energy reserve in yeast cells. It will be liberated from triacylglycerol and oxidized in peroxisomes when cells enter the stationary phase. Fatty acid oxidation requires plenty of $\mathrm{NAD}^{+}$as a coenzyme which is continually supplied by Mdh3p and Gpd1p activity in peroxisomes (Figure 1a). Mdh3p is malate dehydrogenase localizing in peroxisomes and Gpd1p is glycerol 3-phosphate dehydrogenase localizing in both cytosolic and peroxisomal compartments [28]. The substrate for Mdh3p is oxaloacetate derived from aspartate transamination which is replenished by Agc1p activity (Figure 1a). As seen in Figure 1b, deletion of AGC1 decreased fat utilization in the stationary phase, but deletion of GPD1 decreased both of fat storage and fat utilization. Interestingly, deletion of $A G C 1$ in gpd1 1 yeasts restored the capability of fat storage and fat utilization in the stationary phase.

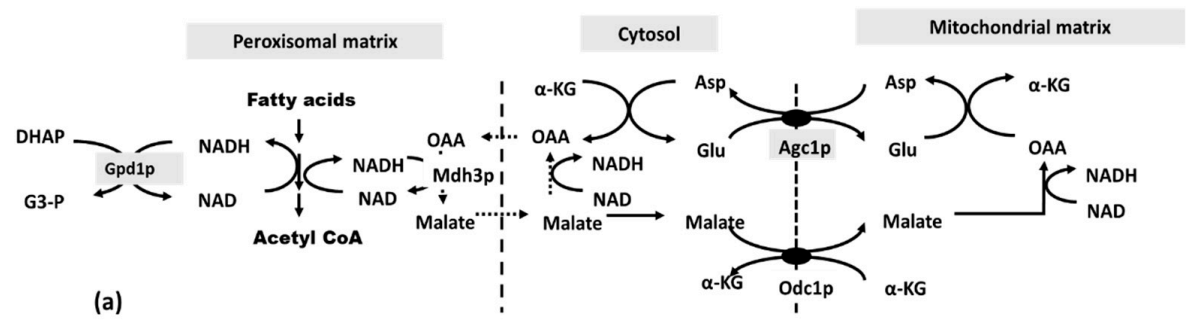

(a)

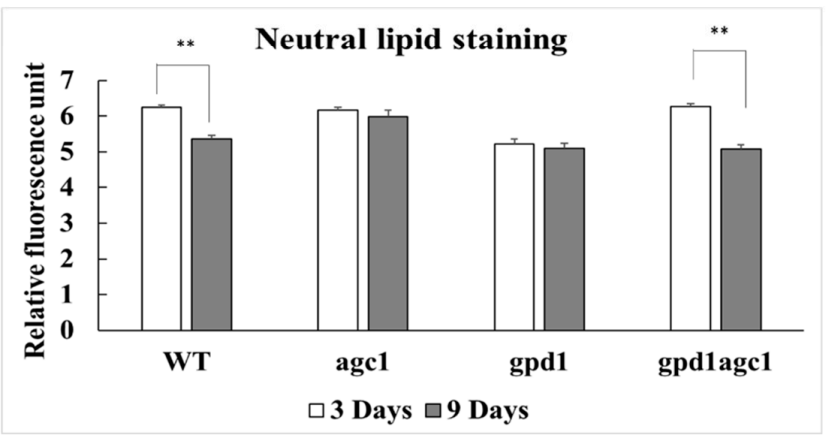

Figure 1. Role of mitochondrial aspartate-glutamate carrier (Agc1p) in the malate-aspartate shuttle involved in fatty acid oxidation in peroxisomes and alteration of fat utilization of agc1 $\Delta$ yeasts and related yeast mutants in the stationary phase. (a) The malate-aspartate shuttle in yeasts is required for $\mathrm{NAD}^{+}$regeneration in peroxisomes with the transfer of the reducing equivalent from peroxisome into mitochondria. Reducing equivalents generated in peroxisomes are derived from fatty acid oxidation which is enhanced by Mdh3p-mediated and Gpd1p-mediated NAD ${ }^{+}$regeneration in peroxisomes. Note that both the malate-oxaloacetate NADH shuttle (dot line) and the malate-aspartate shuttle are linked for $\mathrm{NAD}^{+}$regeneration in peroxisomes. (b) Wild-type and yeast mutants including agc1 $\Delta$, gpd1 1 , and $g p d 1 \Delta a g c 1 \Delta$ cells in stationary phase were selected for neutral lipid staining with Nile red fluorescence dye and then the fluorescence intensity was measured with spectrofluorometry. Note that deletion of AGC1 decreased fat utilization, but deletion of GPD1 decreased both of fat storage and utilization in the stationary phase. Deletion of $A G C 1$ in gpd1 1 yeasts restored fat storage and fat utilization in the stationary phase. Abbreviations: Asp, aspartate; Glu, glutamate; OAA, oxaloacetate; 
$\alpha-K G, \alpha$-ketoglutarate, Agc1p, aspartate-glutamate carrier, Odc1p, oxodicarboxylate carrier; Mdh3p, malate dehydrogenase3; Gpd1p, glycerol 3-phosphate dehydrogenase; DHAP, dihydroxyacetone phosphate; G3-P, glycerol 3-phosphate dehydrogenase. ${ }^{* *} p \leq 0.01$.

3.2. Deletion of AGC1 Increases Peroxisomal NADH Signals, but Decreases Cytosolic NADH Signals in the Stationary Phase

$\mathrm{NAD}^{+}$regeneration in peroxisomes supplies $\mathrm{NAD}^{+}$for fatty acid oxidation. To further explain whether impaired fat utilization in $a g c 1 \Delta$ yeasts is due to decreased $\mathrm{NAD}^{+}$regeneration in peroxisomes, we demonstrated peroxisomal NADH signals in yeasts by peroxisomal NADH sensor plasmids. As shown in Figure 2, increased peroxisomal NADH signals, which are represented as small punctate green fluorescence signals, were observed in $a g c 1 \Delta, g p d 1 \Delta$, and $g p d 1 \Delta a g c 1 \Delta$ yeasts. Deletion of GPD1 showed slightly higher peroxisomal NADH signal intensity relative to others. However, some NADH signals induced by peroxisome NADH sensor plasmids were also found in the cytoplasm. In addition, we used cytosolic NADH sensor plasmids to directly demonstrate cytosolic NADH signals which might be affected in $a g c 1 \Delta$ and $g p d 1 \Delta$ yeasts. The results showed that the cytosolic NADH signals were decreased in $a g c 1 \Delta$ and $g p d 1 \Delta$ yeasts, but these signals were restored in $g p d 1 \Delta a g c 1 \Delta$ yeasts. As seen in Figure 2, some NADH signals induced by cytosolic NADH sensor plasmids were also observed in peroxisomes.

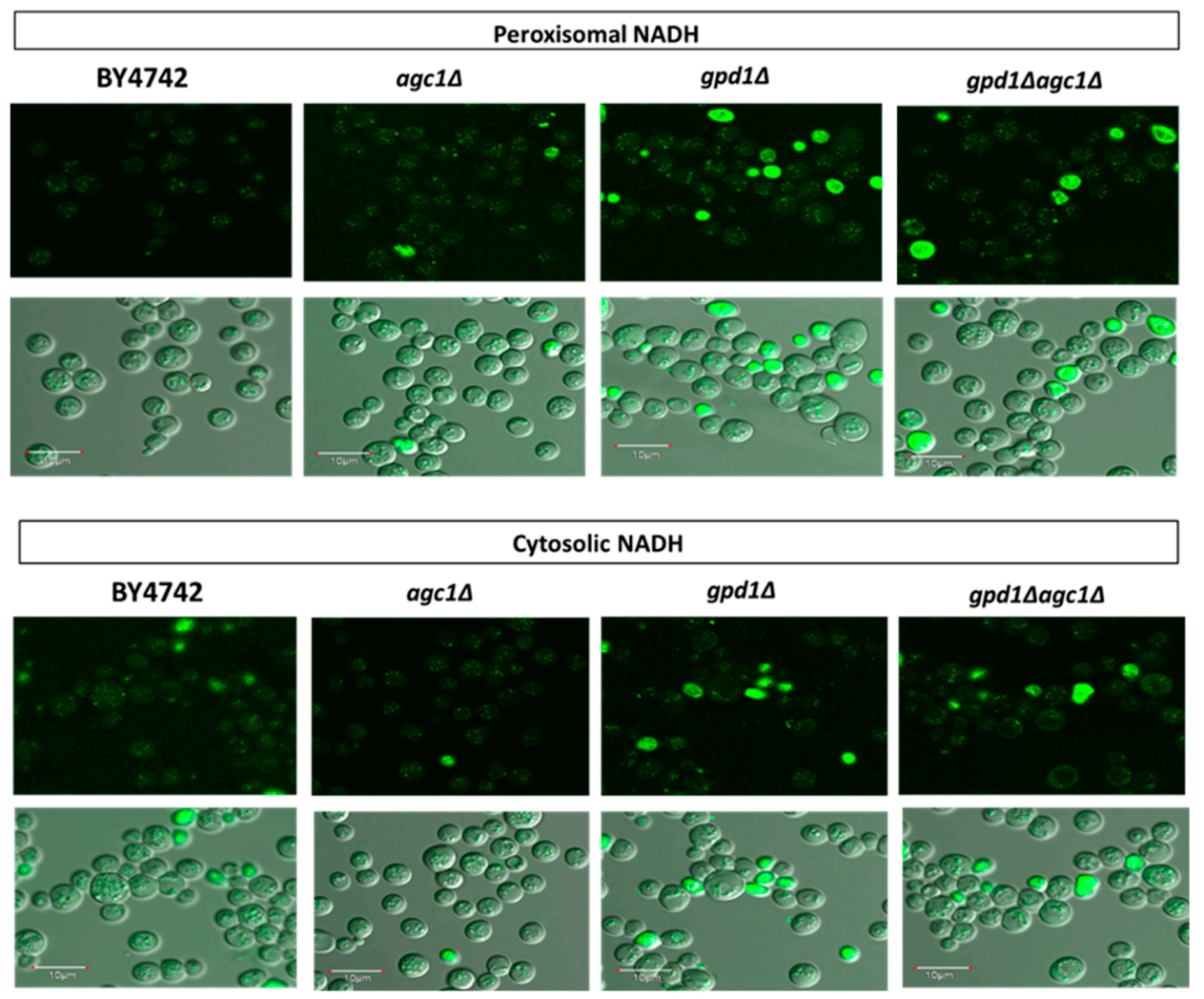

Figure 2. Deletion of $A G C 1$ increases peroxisomal NADH signals but decreases cytosolic NADH signals in the stationary phase. Yeasts containing peroxisomal and cytosolic NADH sensor plasmids were grown in a synthetic deficient (SD) medium plate supplemented with $2 \%$ glucose for five days and then taken for visualizing NADH signals with confocal fluorescence microscopy. The small punctate green fluorescence signals represented NADH localizing in peroxisomes. Note that deletion of GPD1 showed similar effects as deletion of $A G C 1$, and deletion of $A G C 1$ in gpd1 $\triangle$ cells also restored cytosolic $\mathrm{NADH}$ signals. In addition, cytosolic NADH signals induced by peroxisomal NADH sensor plasmids and peroxisomal NADH signals induced by cytosolic NADH sensor plasmids were observed. 


\subsection{Over-Expression of PEX34, MDH3, GPD1 Increases the Longevity of agc1 1 Yeasts in the Stationary Phase}

Peroxisome functions are important for the stationary phase of cell growth, and deletion of ACG1 impairs utilization of acetate and oleic acid, which are important carbon sources in the stationary phase $[12,17,19]$. Peroxisome biogenesis requires both proliferation of the peroxisome structure and import of peroxisomal proteins. PEX34 and PEX11 function in peroxisome proliferation [16]. In contrast, PEX5 functions in the import of peroxisome matrix proteins, such as Mdh3p, which is important for $\mathrm{NAD}^{+}$regeneration in peroxisomes $[16,28]$.

As seen in Figure 3, deletion of AGC1 decreased the chronological lifespan of yeast cells. Over-expression of Pex34p, Pex5p, and Mdh3p in agc1 $\Delta$ cells increased lifespan, but the over-expression of Pex34p, and Pex11p increased longevity in wild-type cells. Interestingly, over-expression of Gpd1 in $a g c 1 \Delta$ cells also increased the chronological lifespan and over-expression of Nde2p, which is an external NADH dehydrogenase in the mitochondria required for catalyzing NADH in cytosol [29], failed to restore the chronological lifespan of agc1 $\Delta$ cells. Conversely, over-expression of $\mathrm{Nde} 2 \mathrm{p}$ and Gpd1p increased the chronological lifespan of wild-type yeasts.

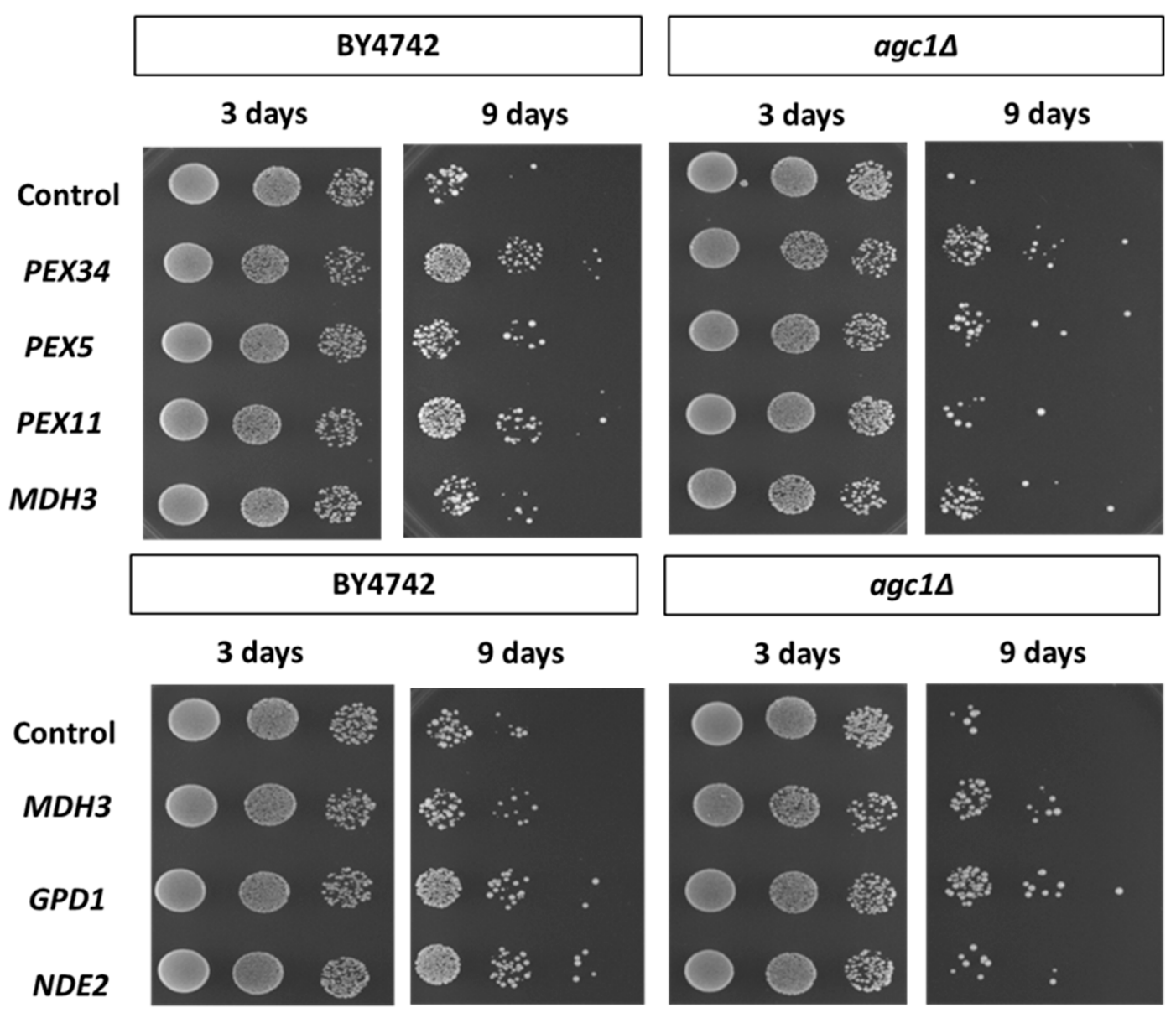

Figure 3. Effects of over-expression of peroxisomal genes and $\mathrm{NAD}^{+}$regeneration genes on the chronological longevity of $a g c 1 \Delta$ yeasts. Wild-type and $\operatorname{agc1\Delta }$ yeasts containing expression plasmids of peroxisomal genes and $\mathrm{NAD}^{+}$regeneration genes as indicated were cultured in SD broth supplemented with $2 \%$ glucose and tested for chronological longevity. The yeast array on an SD plate supplemented with $2 \%$ glucose indicated viability starting with $10^{5}, 10^{4}$, and $10^{3}$ cells. Note that agc1 $\triangle$ yeasts had decreased chronological longevity, and GPD1, MDH3, and PEX34 over-expression increased the longevity of $a g c 1 \Delta$ yeasts. 


\subsection{PEX34-Mediated Chronological Longevity Requires GPD1 but not PEX25 and PEX27}

We have known that the induction of $\mathrm{NAD}^{+}$regeneration in peroxisomes was a primary mechanism of chronological longevity in $a g c 1 \Delta$ yeasts. To determine whether $M D H 3$ was important for increased longevity from over-expression of PEX34, we examined the lifespan of an $a g c 1 \Delta m d h 3 \Delta$ strain. The increased chronological lifespan in $a g c 1 \Delta$ cells from $P E X 34$ over-expression was still apparent, although reduced, when $M D H 3$ was also deleted (Figure 4). This indicates that while over-expression of $M D H 3$ can extend yeast lifespan, this gene does not have a major role in lifespan extension from PEX34 over-expression. In addition, we determined whether GPD1 was important for promoting longevity in agc1 1 yeasts. Interestingly, PEX34 over-expression in the gpd1 $\Delta$ strain caused decreased longevity; however, the negative effect of PEX34 was abolished in the agc1 $g$ gd1 $\Delta$ strain (Figure 4). Unlike the parental agc1 $\Delta$ strain, PEX34 over-expression was unable to substantially increase longevity when GPD1 was deleted. This observation is consistent with GPD1 participating in the PEX34-mediated extension of lifespan in $\operatorname{agc} 1 \Delta$ cells.

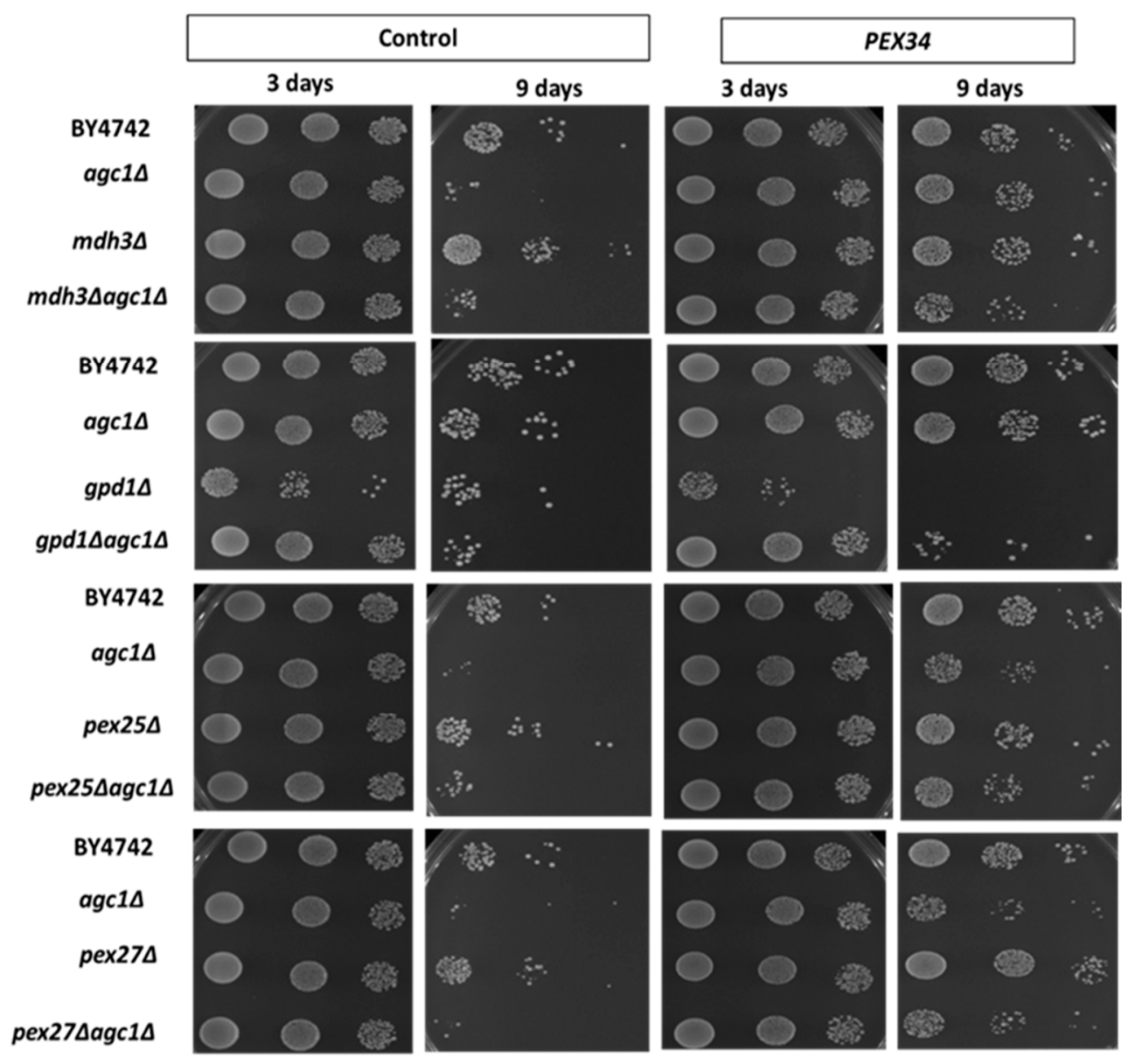

Figure 4. PEX34-mediated longevity requires peroxisomal genes for $\mathrm{NAD}^{+}$regeneration, but not for peroxisome proliferation. Yeasts lacking genes involved in $\mathrm{NAD}^{+}$regeneration in the peroxisome (MDH3, GPD1) and peroxisome proliferation (PEX25, PEX27) were tested for chronological longevity under PEX34 over-expression. The yeast array on an SD plate supplemented with $2 \%$ glucose indicated viability starting with $10^{5}, 10^{4}$, and $10^{3}$ cells. Note that PEX34-mediated longevity requires GPD1 expression and loss of GPD1 decreased the longevity at the early stationary phase.

We also investigated whether the induction of peroxisome proliferation was involved in PEX34-mediated longevity in agc1 $\triangle$ yeast. PEX25 and PEX27 encode peroxisome membrane proteins that physically interact with Pex34p and are required for PEX34-induced peroxisome proliferation [30]. 
We observed that the PEX34-mediated longevity of wild-type and agc1 $\triangle$ yeasts was not reduced in the absence of either PEX25 or PEX27 (Figure 4). In addition, we investigated peroxisome numbers under PEX34 over-expression in limited glucose conditions and found that PEX34 induced peroxisome proliferation in both wild-type and agc1 1 yeasts. It was noticed that PEX34 over-expression also induced the proliferation of tiny peroxisomes (Figure 5). Moreover, PEX34 partially restored the number of peroxisomes in pex $25 \Delta$ yeasts but failed to restore the number of peroxisomes in pex $27 \Delta$ yeasts (Figure 5). These data confirm that the induction of peroxisome proliferation is not a primary mechanism for PEX34-mediated longevity.

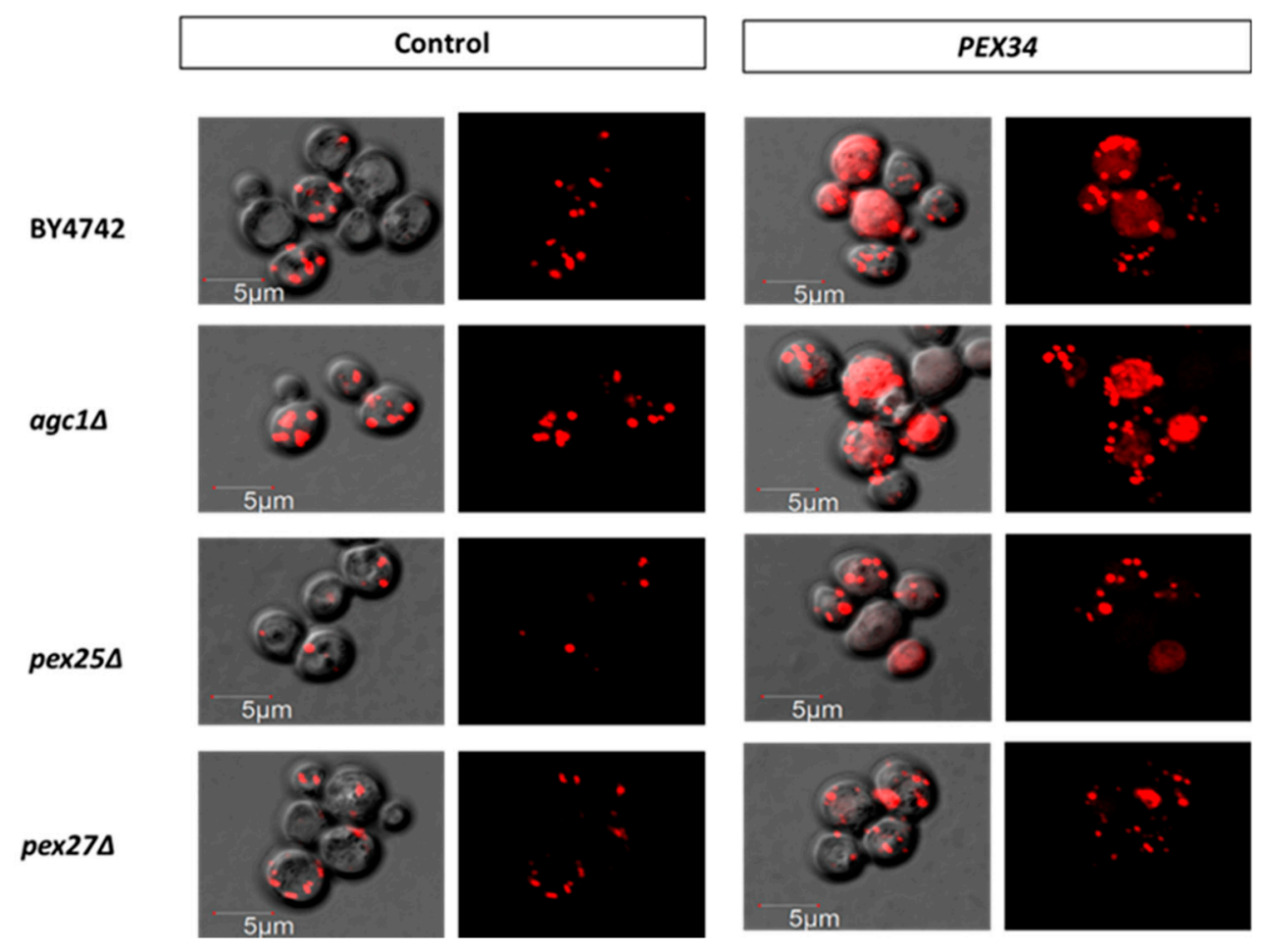

Figure 5. PEX34-induced peroxisome proliferation requires PEX27 under a limited glucose condition. Yeasts lacking genes involved in peroxisome proliferation (PEX25, PEX27) containing peroxisome marker plasmid (RFP-PTS1) were tested for visualizing peroxisomes under PEX34 over-expression. Yeasts were grown in SD broth supplemented with $0.1 \%$ glucose until late log phase and then used for imaging peroxisomes with confocal fluorescence microscopy. The red-dot signal represented peroxisomes. Note that PEX34 over-expression induced the proliferation of tiny peroxisomes.

\subsection{Role of Malate-Oxaloacetate NADH Perosixomal shuttle in Chronological Longevity of agc1 $\mathrm{Y}$ Yeast}

We investigated whether disruption of the malate-oxaloacetate NADH shuttle was sufficient to induce longevity of agc1 $\Delta$ cells. CIT2 encodes a peroxisomal citrate synthase that catalyzes the conversion of oxaloacetate and acetyl CoA to citrate and coenzyme A. ODC1 encodes a mitochondrial oxodicarboxylate carrier, mediating exchange transport of malate and $\alpha$-ketoglutarate between the cytosol and mitochondria [31,32]. It has been reported that CIT2 and ODC1 activity disrupt the malate-oxaloacetate NADH peroxisomal shuttle [32,33]. As seen in Figure 6, deletion of either CIT2 or ODC1 increased the lifespan of $a g c 1 \Delta$ yeast.

Mdh3p and Mdh2p enzymes are components of the peroxisomal malate-oxaloacetate NADH shuttle [28]. We have examined that PEX34-mediated chronological longevity required GPD1 for NAD ${ }^{+}$ regeneration in peroxisomes. However, deletion of $M D H 3$ slightly decreased the PEX34-mediated chronological longevity of agc1 $\Delta$ yeasts (Figure 4). Interestingly, PEX34 over-expression was capable of increasing longevity in the $a g c 1 \Delta m d h 2 \Delta$ strain (Figure 6). This indicates that $M D H 2$ is not required 
for PEX34-mediated longevity in agc1 $\Delta$ cells and is consistent with increased longevity from PEX34 over-expression from GPD1-mediated $\mathrm{NAD}^{+}$regeneration in peroxisomes without flux through the malate-oxaloacetate NADH shuttle (Figure 7).

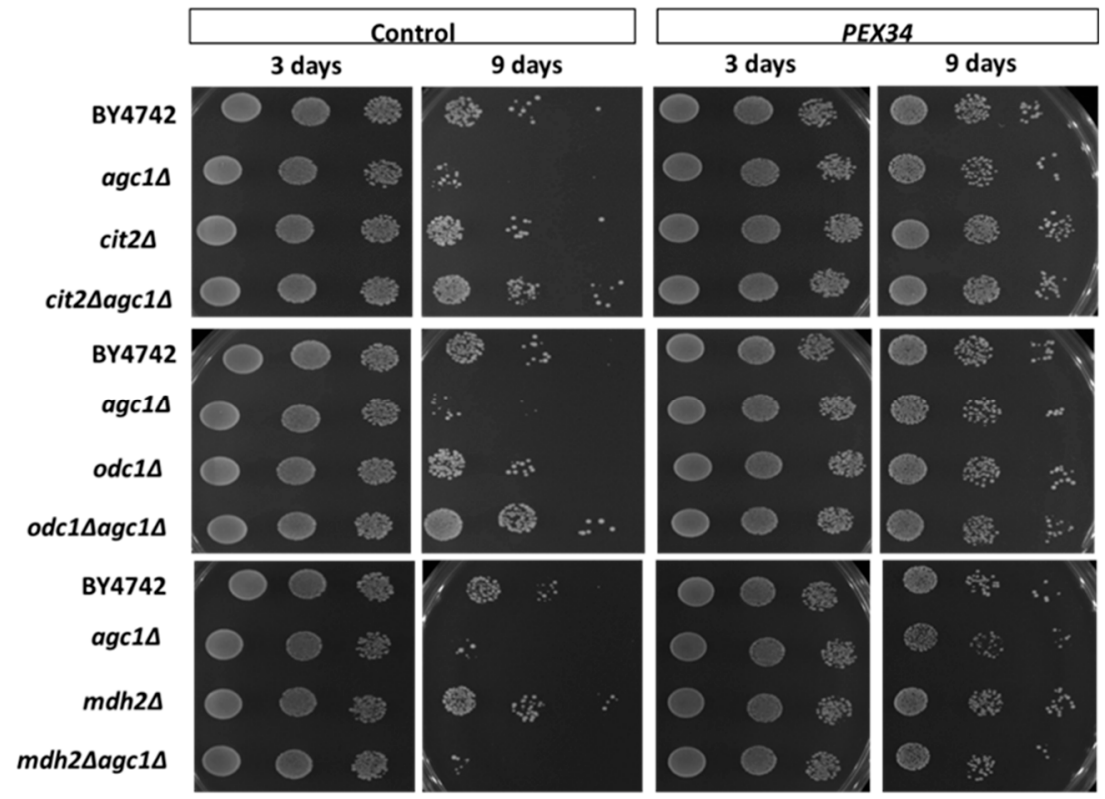

Figure 6. Activation of the malate-oxaloacetate NADH peroxisomal shuttle is sufficient for inducing longevity of $a g c 1 \Delta$ yeasts, but the malate-oxaloacetate NADH peroxisomal shuttle is NOT essential for PEX34-mediated longevity in yeasts. Yeasts lacking genes disrupting the peroxisomal malate-oxaloacetate NADH shuttle $(C I T 2, O D C 1)$ and genes required for this shuttle $(M D H 2)$ were tested for cell viability during the stationary phase under PEX34 over-expression. The yeast array on an SD plate supplemented with $2 \%$ glucose indicated viability starting with $10^{5}, 10^{4}$, and $10^{3}$ cells.

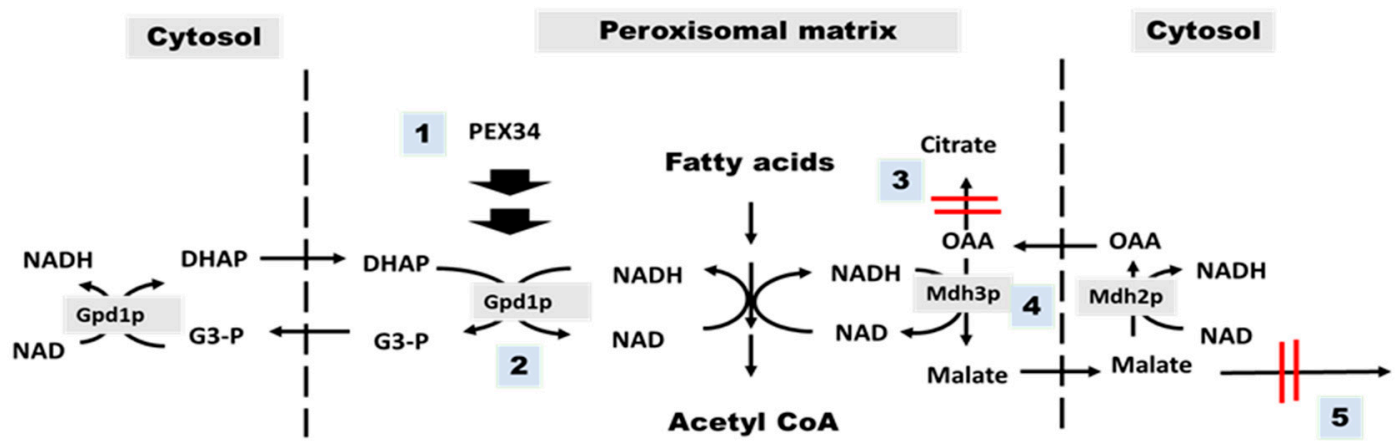

Figure 7. Summary of genetic mechanisms to restore the chronological longevity of agc1 $\Delta$ yeasts. There are five genetic mechanisms for restoring the longevity of agc1 $\Delta$ yeasts, including activation of PEX34 over-expression (1), GPD1 over-expression (2), deletion of CIT2 (3), MDH3 over-expression (4), and deletion of ODC1 (5). The principle of the genetic mechanism for restoring longevity of $\operatorname{agc} 1 \triangle$ yeasts is that induction of GPD1-mediated NAD regeneration in peroxisomes $(\mathbf{1}, \mathbf{2})$ and enhanced peroxisomal malate-oxaloacetate NADH shuttle activity (3-5). Abbreviations: Asp, aspartate; Glu, glutamate; OAA, oxaloacetate; $\alpha-\mathrm{KG}, \alpha$-ketoglutarate, Agc1p, aspartate-glutamate carrier, Odc1p, oxodicarboxylate carrier; Mdh3p, malate dehydrogenase3; Mdh2p, malate dehydrogenase2; Cit2p, citrate synthase2; Gpd1p, glycerol 3-phosphate dehydrogenase 1; DHAP, dihydroxyacetone phosphate; G3-P, glycerol 3-phosphate. 


\section{Discussion}

The models previously used to study the pathogenesis of citrin deficiency were citrin/mitochondrial glycerol 3-phosphate dehydrogenase (mGPD) double-knockout mice and patients' hepatocytes $[1,34]$. In this study, we firstly proposed $\operatorname{agc}_{\mathrm{g}} 1 \Delta$ yeasts to be used as a model organism to study the pathogenesis of citrin deficiency and investigated the effects of peroxisomal genes on the longevity of $a g c 1 \Delta$ yeasts. The advantage of yeast in this study is its short lifespan through which we can observe chronological longevity. Moreover, yeasts contain haploid chromosomes which can easily be genetically manipulated to suit our experimental design. Although, yeasts are evolutionarily diverse from human hepatocytes and some peroxisomal genes are specific in yeast species, the basic functions in yeasts, such as fatty acid oxidation, $\mathrm{NAD}^{+}$regeneration, and peroxisome proliferation, also exist in human hepatocytes. Therefore, the information from this study can provide some points to further study in citrin deficiency patients.

In our study, deletion of $A G C 1$, encoding the mitochondrial aspartate-glutamate carrier, results in decreased fat utilization, decreased cytosolic NADH, and increased peroxisomal NADH signals in the stationary phase. These data suggest that loss of $A G C 1$ causes impaired $\mathrm{NAD}^{+}$regeneration in peroxisomes resulting in decreased fatty acid oxidation with reduced production of reducing equivalents in the cytosol. These results are similar to the deletion of GPD1, encoding glycerol 3-phosphate dehydrogenase required for $\mathrm{NAD}^{+}$regeneration in peroxisomes. Moreover, the deletion of GPD1 also impaired fat storage at the early stationary phase. As known, Gpd1p is also required for production of glycerol, which plays role in triacylglycerol synthesis, so loss of Gpd1p results in decreased fat storage. Interestingly, deletion of AGC1 in gpd1 $\Delta$ cells could restore fat storage, fat utilization, and correct NADH balance in the cytosol by unknown mechanisms, which are being further investigated. Moreover, the deletion of $A G C 1$ results in decreased lifespan in yeasts and over-expression of GPD1 or $M D H 3$, which directly enhances NAD regeneration in peroxisomes and could restore the longevity of agc1 $\Delta$ cells. Moreover, enhanced activity of the malate-oxaloacetate NADH peroxisomal shuttle, due to deletion of CIT2 and ODC1, could restore or even enhance the longevity of $a g c 1 \Delta$ cells. Collectively, it confirmed that the impaired $\mathrm{NAD}^{+}$regeneration in peroxisomes is a cause of decreased chronological lifespan of agc1 $\Delta$ cells.

PEX34 over-expression can induce chronological longevity of $a g c 1 \Delta$ as well as wild-type yeasts. $P E X 34$ is required for peroxisome proliferation, and over-expression of PEX34 induces peroxisome proliferation in oleic medium, through a process that is dependent on Pex25p and Pex27p [30]. However, PEX34-mediated increased longevity was independent of the presence of PEX25 and PEX27, but PEX34-mediated peroxisome proliferation required the presence of PEX27 under a limited glucose condition. This suggests that peroxisomal proliferation is not the primary mechanism involved in promoting increased lifespan from PEX34 over-expression. Consistent with this proposal, over-expression of PEX11, which can induce peroxisome proliferation in oleic medium [13], did not increase the longevity of agc1 $\Delta$ cells. Interestingly, we demonstrated that PEX34-mediated longevity needs GPD1 expression. The ability of PEX34 to promote longevity in $a g c 1 \triangle$ cells was observed in the absence of $M D H 2$ and $M D H 3$, required for the peroxisomal malate-oxaloacetate NADH shuttle. This suggests that another mechanism is involved in GPD1-dependent lifespan extension from PEX34 over-expression. Moreover, over-expression of GPD1 itself increases the chronological lifespan of both wild-type and $a g c 1 \Delta$ cells, but over-expression of $M D H 3$ increases the chronological lifespan in $a g c 1 \Delta$ cells only, indicating that the GPD1-induced longevity mechanisms are not specific and may induce other mechanisms with $\mathrm{NAD}^{+}$regeneration in peroxisomes. Gpd1p is required for glycerol production, which can protect cells under several stress conditions, such as osmotic stress, oxidative stress, or ER stress [35,36]. Our group has previously shown that PEX34 over-expression results in aberrant ER morphology and induces ER stress in an acetate medium [37]. It is possible that PEX34 over-expression also induces ER stress in the stationary phase of glucose-grown cells, resulting in the induction of GPD1-mediated $\mathrm{NAD}^{+}$regeneration in peroxisomes. Enhanced chronological lifespan can also be mediated by the activation of mitochondrial retrograde and oxidative stress responses to overcome 
mitochondrial stresses $[38,39]$. We have previously shown that PEX34 over-expression induced mitochondrial retrograde signaling in acetate medium [37], suggesting that PEX34 over-expression can induce these stress responses resulting in increased longevity in yeasts.

Citrin deficiency results in intrahepatic cholestasis and fatty liver. The mechanisms of intrahepatic cholestasis and fatty liver in citrin deficiency might be involved in impaired bile acid conjugation and impaired fatty acid oxidation, which requires $\mathrm{NAD}^{+}$regeneration in peroxisomes $[1,7,40,41]$. Administration of sodium pyruvate has the potential to increase $\mathrm{NAD}^{+}$regeneration in peroxisomes, due to the presence of lactate dehydrogenases in both peroxisome and cytosolic compartments in human cells [42]. Moreover, current evidence revealed malate dehydrogenase in hepatic peroxisomes [42], indicating the presence of a peroxisomal malate-oxaloacetate NADH shuttle in humans. Based on our findings, activation the hepatic malate-oxaloacetate NADH shuttle may reduce the symptoms of citrin deficiency. Moreover, activation of peroxisome proliferator-activated receptor-alpha (PPAR $\alpha)$ induces fatty acid oxidation in both the peroxisome and mitochondria [43]. Administration of the PPAR $\alpha$ drug in humans might increase $\mathrm{NAD}^{+}$regeneration in peroxisomes and decrease fatty liver in citrin-deficient patients, as well as restore cellular lifespan to levels seen in normal cells.

\section{Conclusions}

Peroxisomal dysfunctions observed using the yeast model for citrin deficiency appear to result in decreased cellular longevity. Processes that enhance peroxisomal NAD ${ }^{+}$regeneration, either directly or indirectly, can increase chronological lifespan in $a g c 1 \Delta$ yeast (Figure 7). Enhancing peroxisome function or inducing $\mathrm{NAD}^{+}$regeneration in peroxisomes are suggested for further study in patients' hepatocytes.

Supplementary Materials: The following are available online at http://www.mdpi.com/2079-9721/8/1/2/s1, Table S1: Primer names and their sequences used in this study, Table S2: Yeast genotypes used in this study.

Author Contributions: Conceptualization, C.C. and L.T.J.; methodology, C.C.; validation, C.C. and T.T.; formal analysis, C.C.; investigation, C.C. and T.T.; resources, L.T.J.; data curation, C.C.; writing-original draft preparation, C.C.; writing - review and editing, C.C., D.W., and L.T.J.; visualization, C.C.; supervision, D.W. and L.T.J.; project administration, C.C.; funding acquisition, C.C., D.W., and L.T.J. All authors have read and agreed to the published version of the manuscript.

Funding: This research was funded by a grant from the Mahidol University (to D.W.), a joint grant from the Faculty of Science and Faculty of Medicine, Ramathibodi Hospital, Mahidol University (to L.J. and D.W.), a grant from the Faculty of Medicine, Khon Kaen University (to C.C.), and a grant from the Thailand Research Fund IRG5980008. The article processing charge (APC) was paid by C.C.

Acknowledgments: The authors gratefully acknowledge all L.J.'s lab members for technical support.

Conflicts of Interest: The authors declare no conflict of interest.

\section{References}

1. Komatsu, M.; Kimura, T.; Yazaki, M.; Tanaka, N.; Yang, Y.; Nakajima, T.; Horiuchi, A.; Fang, Z.Z.; Joshita, S.; Matsumoto, A.; et al. Steatogenesis in adult-onset type II citrullinemia is associated with down-regulation of PPARalpha. Biochim. Biophys. Acta 2015, 1852, 473-481. [CrossRef]

2. Shigematsu, Y.; Hirano, S.; Hata, I.; Tanaka, Y.; Sudo, M.; Sakura, N.; Tajima, T.; Yamaguchi, S. Newborn mass screening and selective screening using electrospray tandem mass spectrometry in Japan. J. Chromatogr. B 2002, 776, 39-48. [CrossRef]

3. Wongkittichote, P.; Sukasem, C.; Kikuchi, A.; Aekplakorn, W.; Jensen, L.T.; Kure, S.; Wattanasirichaigoon, D. Screening of SLC25A13 mutation in the Thai population. World J. Gastroenterol. 2013, 19, 7735-7742. [CrossRef]

4. Kobayashi, K.; Sinasac, D.S.; Iijima, M.; Boright, A.P.; Begum, L.; Lee, J.R.; Yasuda, T.; Ikeda, S.; Hirano, R.; Terazono, H.; et al. The gene mutated in adult-onset type II citrullinaemia encodes a putative mitochondrial carrier protein. Nat. Genet. 1999, 22, 159-163. [CrossRef]

5. Palmieri, L.; Pardo, B.; Lasorsa, F.M.; del Arco, A.; Kobayashi, K.; Iijima, M.; Runswick, M.J.; Walker, J.E.; Saheki, T.; Satrustegui, J.; et al. Citrin and aralar1 are $\mathrm{Ca}(2+)$-stimulated aspartate/glutamate transporters in mitochondria. The EMBO J. 2001, 20, 5060-5069. [CrossRef] 
6. Saheki, T.; Kobayashi, K.; Iijima, M.; Horiuchi, M.; Begum, L.; Jalil, M.A.; Li, M.X.; Lu, Y.B.; Ushikai, M.; Tabata, A.; et al. Adult-onset type II citrullinemia and idiopathic neonatal hepatitis caused by citrin deficiency: Involvement of the aspartate glutamate carrier for urea synthesis and maintenance of the urea cycle. Mol. Genet. Metab. 2004, 81, 20-26. [CrossRef]

7. Saheki, T.; Song, Y.Z. Citrin Deficiency. In GeneReviews ${ }^{\circledR}$; Adam, M.P., Ardinger, H.H., Pagon, R.A., Wallace, S.E., Bean, L.J.H., Stephens, K., Amemiya, A., Eds.; University of Washington: Seattle, Seattle, WA, USA, 2017.

8. Mutoh, K.; Kurokawa, K.; Kobayashi, K.; Saheki, T. Treatment of a citrin-deficient patient at the early stage of adult-onset type II citrullinaemia with arginine and sodium pyruvate. J. Inherit. Metab. Dis. 2008, 31, 343-347. [CrossRef]

9. Tenreiro, S.; Outeiro, T.F. Simple is good: Yeast models of neurodegeneration. FEMS Yeast Res. 2010, 10, 970-979. [CrossRef]

10. Wongkittichote, P.; Tungpradabkul, S.; Wattanasirichaigoon, D.; Jensen, L.T. Prediction of the functional effect of novel SLC25A13 variants using a S. cerevisiae model of AGC2 deficiency. J. Inherit. Metab. Dis. 2013, 36, 821-830. [CrossRef]

11. Forsburg, S.L. The art and design of genetic screens: Yeast. Nat. Rev. Genet. 2001, 2, 659-668. [CrossRef]

12. Cavero, S.; Vozza, A.; del Arco, A.; Palmieri, L.; Villa, A.; Blanco, E.; Runswick, M.J.; Walker, J.E.; Cerdan, S.; Palmieri, F.; et al. Identification and metabolic role of the mitochondrial aspartate-glutamate transporter in Saccharomyces cerevisiae. Mol. Microbiol. 2003, 50, 1257-1269. [CrossRef] [PubMed]

13. Huber, A.; Koch, J.; Kragler, F.; Brocard, C.; Hartig, A. A subtle interplay between three Pex11 proteins shapes de novo formation and fission of peroxisomes. Traffic 2012, 13, 157-167. [CrossRef]

14. Lee, Y.J.; Jang, J.W.; Kim, K.J.; Maeng, P.J. TCA cycle-independent acetate metabolism via the glyoxylate cycle in Saccharomyces cerevisiae. Yeast 2011, 28, 153-166. [CrossRef] [PubMed]

15. Pascual-Ahuir, A.; Manzanares-Estreder, S.; Proft, M. Pro- and Antioxidant Functions of the Peroxisome-Mitochondria Connection and Its Impact on Aging and Disease. Oxidative Med. Cell. Longev. 2017, 2017, 9860841. [CrossRef]

16. Smith, J.J.; Aitchison, J.D. Peroxisomes take shape. Nat. Rev. Mol. Cell Boil. 2013, 14, 803-817. [CrossRef]

17. Lefevre, S.D.; van Roermund, C.W.; Wanders, R.J.; Veenhuis, M.; van der Klei, I.J. The significance of peroxisome function in chronological aging of Saccharomyces cerevisiae. Aging Cell 2013, 12, 784-793. [CrossRef]

18. van Roermund, C.W.; Waterham, H.R.; Ijlst, L.; Wanders, R.J. Fatty acid metabolism in Saccharomyces cerevisiae. Cell. Mol. Life Sci. CMLS 2003, 60, 1838-1851. [CrossRef]

19. Longo, V.D.; Shadel, G.S.; Kaeberlein, M.; Kennedy, B. Replicative and chronological aging in Saccharomyces cerevisiae. Cell Metab. 2012, 16, 18-31. [CrossRef]

20. Sikorski, R.S.; Hieter, P. A system of shuttle vectors and yeast host strains designed for efficient manipulation of DNA in Saccharomyces cerevisiae. Genetics 1989, 122, 19-27.

21. Sullivan, J.A.; Lewis, M.J.; Nikko, E.; Pelham, H.R. Multiple interactions drive adaptor-mediated recruitment of the ubiquitin ligase rsp5 to membrane proteins in vivo and in vitro. Mol. Biol. Cell 2007, 18, 2429-2440. [CrossRef]

22. Bilan, D.S.; Matlashov, M.E.; Gorokhovatsky, A.Y.; Schultz, C.; Enikolopov, G.; Belousov, V.V. Genetically encoded fluorescent indicator for imaging $\mathrm{NAD}(+) / \mathrm{NADH}$ ratio changes in different cellular compartments. Biochim. Biophys. Acta 2014, 1840, 951-957. [CrossRef]

23. Brachmann, C.B.; Davies, A.; Cost, G.J.; Caputo, E.; Li, J.; Hieter, P.; Boeke, J.D. Designer deletion strains derived from Saccharomyces cerevisiae S288C: A useful set of strains and plasmids for PCR-mediated gene disruption and other applications. Yeast 1998, 14, 115-132. [CrossRef]

24. Longtine, M.S.; McKenzie, A., 3rd; Demarini, D.J.; Shah, N.G.; Wach, A.; Brachat, A.; Philippsen, P.; Pringle, J.R. Additional modules for versatile and economical PCR-based gene deletion and modification in Saccharomyces cerevisiae. Yeast 1998, 14, 953-961. [CrossRef]

25. Gietz, R.D.; Schiestl, R.H. Applications of high efficiency lithium acetate transformation of intact yeast cells using single-stranded nucleic acids as carrier. Yeast 1991, 7, 253-263. [CrossRef]

26. Sherman, F.; Fink, G.R.; Lawrence, C.W. Methods in Yeast Genetics; Cold Spring Harbor Laboratory Press: Cold Spring Harbor, NY, USA, 1978; pp. 178-179. 
27. Zipor, G.; Haim-Vilmovsky, L.; Gelin-Licht, R.; Gadir, N.; Brocard, C.; Gerst, J.E. Localization of mRNAs coding for peroxisomal proteins in the yeast, Saccharomyces cerevisiae. Proc. National Acad. Sci. USA 2009, 106, 19848-19853. [CrossRef]

28. Al-Saryi, N.A.; Al-Hejjaj, M.Y.; van Roermund, C.W.T.; Hulmes, G.E.; Ekal, L.; Payton, C.; Wanders, R.J.A.; Hettema, E.H. Two NAD-linked redox shuttles maintain the peroxisomal redox balance in Saccharomyces cerevisiae. Sci. Rep. 2017, 7, 11868. [CrossRef]

29. Luttik, M.A.; Overkamp, K.M.; Kotter, P.; de Vries, S.; van Dijken, J.P.; Pronk, J.T. The Saccharomyces cerevisiae NDE1 and NDE2 genes encode separate mitochondrial NADH dehydrogenases catalyzing the oxidation of cytosolic NADH. J. Biol. Chem. 1998, 273, 24529-24534. [CrossRef]

30. Tower, R.J.; Fagarasanu, A.; Aitchison, J.D.; Rachubinski, R.A. The peroxin Pex34p functions with the Pex11 family of peroxisomal divisional proteins to regulate the peroxisome population in yeast. Mol. Boil. Cell 2011, 22, 1727-1738. [CrossRef]

31. Lewin, A.S.; Hines, V.; Small, G.M. Citrate synthase encoded by the CIT2 gene of Saccharomyces cerevisiae is peroxisomal. Mol. Cell. Boil. 1990, 10, 1399-1405. [CrossRef]

32. Palmieri, L.; Agrimi, G.; Runswick, M.J.; Fearnley, I.M.; Palmieri, F.; Walker, J.E. Identification in Saccharomyces cerevisiae of two isoforms of a novel mitochondrial transporter for 2-oxoadipate and 2-oxoglutarate. J. Biol. Chem. 2001, 276, 1916-1922. [CrossRef]

33. Chen, Y.; Siewers, V.; Nielsen, J. Profiling of cytosolic and peroxisomal acetyl-CoA metabolism in Saccharomyces cerevisiae. PLoS ONE 2012, 7, e42475. [CrossRef]

34. Saheki, T.; Iijima, M.; Li, M.X.; Kobayashi, K.; Horiuchi, M.; Ushikai, M.; Okumura, F.; Meng, X.J.; Inoue, I.; Tajima, A.; et al. Citrin/mitochondrial glycerol-3-phosphate dehydrogenase double knock-out mice recapitulate features of human citrin deficiency. J. Biol. Chem. 2007, 282, 25041-25052. [CrossRef]

35. Kaeberlein, M.; Andalis, A.A.; Fink, G.R.; Guarente, L. High osmolarity extends life span in Saccharomyces cerevisiae by a mechanism related to calorie restriction. Mol. Cell. Boil. 2002, 22, 8056-8066. [CrossRef]

36. Torres-Quiroz, F.; Garcia-Marques, S.; Coria, R.; Randez-Gil, F.; Prieto, J.A. The activity of yeast Hog1 MAPK is required during endoplasmic reticulum stress induced by tunicamycin exposure. J. Biol. Chem. 2010, 285, 20088-20096. [CrossRef]

37. Chalermwat, C.; Thosapornvichai, T.; Wongkittichote, P.; Phillips, J.D.; Cox, J.E.; Jensen, A.N.; Wattanasirichaigoon, D.; Jensen, L.T. Over-expression of the peroxin Pex34p suppresses impaired acetate utilization in yeast lacking the mitochondrial aspartate/glutamate carrier Agc1p. FEMS Yeast Res. 2019, 19, foz078. [CrossRef]

38. Breitenbach, M.; Rinnerthaler, M.; Hartl, J.; Stincone, A.; Vowinckel, J.; Breitenbach-Koller, H.; Ralser, M. Mitochondria in ageing: There is metabolism beyond the ROS. FEMS Yeast Res. 2014, 14, 198-212. [CrossRef]

39. Liu, Z.; Butow, R.A. Mitochondrial retrograde signaling. Annu. Rev. Genet. 2006, 40, 159-185. [CrossRef]

40. Ferdinandusse, S.; Denis, S.; Faust, P.L.; Wanders, R.J. Bile acids: The role of peroxisomes. J. Lipid Res. 2009, 50, 2139-2147. [CrossRef]

41. Yang, C.H.; Chen, C.Y.; Chou, Y.Y.; Chiu, H.C.; Tsai, W.L.; Shiesh, S.C. Bile acid profiles in neonatal intrahepatic cholestasis caused by citrin deficiency. Clin. Chim. Acta 2017, 475, 28-35. [CrossRef]

42. Gronemeyer, T.; Wiese, S.; Ofman, R.; Bunse, C.; Pawlas, M.; Hayen, H.; Eisenacher, M.; Stephan, C.; Meyer, H.E.; Waterham, H.R.; et al. The proteome of human liver peroxisomes: Identification of five new peroxisomal constituents by a label-free quantitative proteomics survey. PLoS ONE 2013, 8, e57395. [CrossRef]

43. Rakhshandehroo, M.; Hooiveld, G.; Muller, M.; Kersten, S. Comparative analysis of gene regulation by the transcription factor PPARalpha between mouse and human. PLoS ONE 2009, 4, e6796. [CrossRef] [PubMed]

(C) 2020 by the authors. Licensee MDPI, Basel, Switzerland. This article is an open access article distributed under the terms and conditions of the Creative Commons Attribution (CC BY) license (http://creativecommons.org/licenses/by/4.0/). 